\title{
Proximity Action theory of superconductive nanostructures
}

\author{
M. A. Skvortsov ${ }^{1}$, A. I. Larkin ${ }^{1,2}$ and M. V. Feigel'man ${ }^{1}$ \\ ${ }^{1}$ L. D. Landau Institute for Theoretical Physics, Moscow 117940, Russia \\ ${ }^{2}$ Theoretical Physics Institute, University of Minnesota, Minneapolis, MN 55455, USA
}

\begin{abstract}
We review a novel approach to the superconductive proximity effect in disordered normal-superconducting (N-S) structures. The method is based on the multicharge Keldysh action and is suitable for the treatment of interaction and fluctuation effects. As an application of the formalism, we study the subgap conductance and noise in two-dimensional N-S systems in the presence of the electron-electron interaction in the Cooper channel. It is shown that singular nature of the interaction correction at large scales leads to a nonmonotonuos temperature, voltage and magnetic field dependence of the Andreev conductance.
\end{abstract}

PACS numbers: 74.40.+k, 74.50.+r, 72.10.Bg

\section{INTRODUCTION}

A superconductor in contact with a normal metal induces Cooper correlations between electrons in the normal region, the phenomenon known as the proximity effect. Its microscopic origin lies in Andreev reflection [1] of an electron into a hole at the normal-metalsuperconducting (N-S) interface. The probability of Andreev reflection and thus the strength of the proximity effect is determined by the transparency of the N-S interface and the nature of electron propagation in the $\mathrm{N}$ part of the structure. Disorder in the normal conductor near the N-S contact was shown theoretically [2] 5 to increase considerably the effective probability of Andreev reflection (see Ref. [6] for a recent review from the experimental viewpoint).

The standard semiclassical theory of N-S conductivity [2 5], based either on the traditional nonequilibrium superconductivity approach [7] or on the scattering formalism [8.5], usually neglects interaction effects in the $\mathrm{N}$ part of the structure. However, in low-dimensional structures, Coulomb interaction in the normal diffusive region gets enhanced [9], which may affect strongly the Andreev conductance and noise.

In this paper we address the effect of interaction between electrons in the normal part of an N-S structure on the charge transport through the system. To study a system with interaction a novel theoretical method should be developed since neither of the above-mentioned approaches can handle interaction corrections. Indeed, the scattering matrix formalism relying on the linear relation between the outgoing and incoming states is $a$ priori a one-particle description. On the other hand, Larkin-Ovchinnikov kinetic equation [7] can be generalized to allow for (at least some part of) interaction corrections, but its practical solution seems hardly possible beyond the first order of perturbation theory in interaction strength [10]11.

An appropriate formalism had been developed in Ref. [12] in the framework of the Keldysh action for dis- ordered superonductors [13]. We start from the fully microscopic Lagrangian describing interacting electrons in the diffusive conductor. Then, successively integrating over electronic degrees of freedom in the normal conductor we end up with the Proximity Action, $S_{\text {prox }}\left[Q_{S}, Q_{N}\right]$, which is a functional of two matrices, $Q_{S}$ and $Q_{N}$, describing the states of the superconductive and external normal terminals of the N-S structure (cf. Fig. 1). Once the form of the Proximity Action is known, one can easily calculate the conductivity of the system, current noise, and, in principle, higher correlators of current and even the full statistics of transmitted charge [14, 15].

The Proximity Action approach bears an obvious analogy with the scattering matrix approach [5, 3] as both describe transport properties in terms of the characteristics of the terminals (stationary-state Green functions of the terminals $Q_{S, N}$ in the former versus asymptotic scattering states in the latter approach). In this respect, the Proximity Action method also shares the logic of Nazarov's circuit theory of Andreev conductance 甮. On the other hand, the Keldysh action approach is a natural generalization of the kinetic equation for dirty superconductors for the case of fluctuating fields. The LarkinOvchinnikov kinetic equation then emerges as a saddle point equation for the Keldysh action [13].

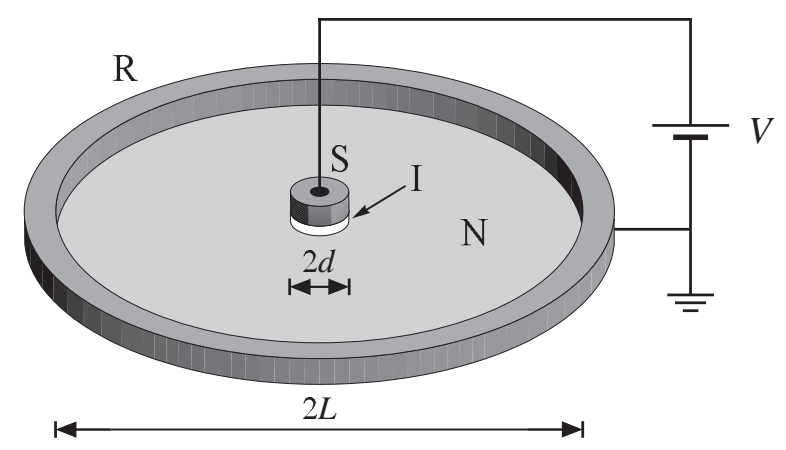

FIG. 1. A small superconductive island (S) of size $2 d$ connected to a reservoir ( $\mathrm{R}$ ) through a tunnel barrier (I) and a dirty normal film $(\mathrm{N})$ of size $2 L \gg 2 d$. 
As an application of the formalism, we will study charge transport in two-dimensional (2D) N-I-S structures shown in Fig. 1 at low (compared to the $S$ gap $\Delta$ ) temperature and voltages, and arbitrary ratio $t=$ $R_{D} / R_{T}$, where $R_{D}$ and $R_{T}$ are the resistances of the diffusive normal conductor, and of the tunnel barrier in the normal state, correspondingly. We will calculate the Andreev conductance and noise of such systems in the presence of Cooper interaction in the normal conductor modified by the Coulomb interaction 16 18, as a function of the "decoherence time" of an electron and the Andreevreflected hole, $\hbar / \Omega_{*}$, where $\Omega_{*}=\max (T, e V, e D H / c)$. We will show that the Cooper interaction effects in $2 \mathrm{D}$ are substantially different from those in the $1 \mathrm{D}$ case considered in Ref. [11]. In particular, the lowest-order relative correction $\delta G_{A} / G_{A}$ scales as $-\lambda \ln (L / d)$ and grows with the size of the system $L$, provided that $\Omega_{*} \ll E_{\mathrm{Th}}=$ $\hbar D / L^{2}$. We will sum up the main logarithmic terms of the order of $[\lambda \ln (L / d)]^{n}$ and find that Cooper repulsion may lead to a nonmonotonuos dependence of $G_{A}$ on $R_{D} / R_{T}$ and on the decoherence energy scale $\Omega_{*}$.

In this paper we will not consider the weak localization [19,20] and interaction-induced corrections [9] to the sheet conductance, $\sigma$, of the normal metal assuming that it is relatively large, $g \equiv\left(\hbar / e^{2}\right) \sigma \gg \ln (L / d)$. We will also not take into account the effect of the Coulomb zerobias anomaly (ZBA) [9,21] on the Andreev conductance. This effect was considered in [13] within the lowest-order tunneling approximation. It was shown, similar to the Coulomb ZBA problem for normal conductors (cf., e. g., Ref. 22]), that it can be strongly suppressed if the bare long-range Coulomb potential is screened, e. g., by placing a nearby metal gate. This is the situation we assume in the present paper.

Another limitation of the present discussion is that we will consider the case of a $2 \mathrm{D}$ geometry of the current flow between the superconductive and normal electrodes of the structure, as shown in Fig. 1. This will make possible to construct a unified functional renormalization group treatment that takes into account modifications of the proximity effect strength both due to multiple Andreev reflections and due to Cooper-channel repulsion. Note that the sample geometry should not necessarily be symmetric as shown in Fig. 1: the only important feature of the geometry chosen is the $2 \mathrm{D}$ spreading of the current flow.

\section{MULTICHARGE PROXIMITY ACTION}

Here we present a brief overview of the Proximity Action approach to charge transport in N-I-S systems elaborated in Ref. [12. Eliminating all dynamic degrees of freedom in the normal diffusive conductor, one reduces the total action of the system to the form $S_{\text {prox }}\left[Q_{S}, Q_{N}\right]$, with the matrices $Q_{S}$ and $Q_{N}$ having the meaning of the steady-state Keldysh Green function of the $\mathrm{S}$ island and external normal terminal labeled by $\mathrm{R}$ in Fig. 1, respectively. In the low frequency limit, $\omega \ll E_{\mathrm{Th}}=D / L^{2}$, when diffusive electron motion spreads over the whole conducting region during one circle of the external voltage, the resulting Proximity Action has the form

$$
S_{\text {prox }}\left[Q_{S}, Q_{N}\right]=-i \pi^{2} g \sum_{n=1}^{\infty} \gamma_{n} \operatorname{Tr}\left(Q_{S} Q_{N}\right)^{n}
$$

The action is known once the infinite set of parameters ("charges") $\gamma_{n}$ is specified.

Remarkably, for noninteracting systems, knowledge of $\left\{\gamma_{n}\right\}$ is equivalent to knowledge of the whole distribution function $\mathcal{P}(T)$ of transmission coefficients of the system. To formulate this analogy, it is convenient to make a Fourier transform from $\left\{\gamma_{n}\right\}$ to a $2 \pi$-periodic function $u(x)$ of an auxiliary continuous variable $x$ :

$$
u(x)=\sum_{n=1}^{\infty} n \gamma_{n} \sin n x .
$$

The relation between the function $u(x)$ and the generating function of transmission coefficients introduced by Nazarov [3] (cf. also Ref. 23) was established in Ref. 12] by direct comparison:

$$
4 \pi g u(x)=\int_{0}^{1} \frac{T \sin x}{1-T \sin ^{2}(x / 2)} \mathcal{P}(T) d T .
$$

For interacting systems and systems with fluctuations [24], Eq. (3) may be interpreted as a generalization of the notion of transmission coefficients.

Determination of $\left\{\gamma_{n}\right\}$ is a separate task related with elimination of fast modes in the normal conductor. We will discussed it later, while now we will explain how to obtain transport properties from the Proximity Action (11). In order to study conducting properties of the system, we apply a bias voltage $V$ to the superconducting and normal terminals of the structure and study its current response. The resulting expressions for the Andreev conductance (in units of $e^{2} / \hbar$ ) and noise power take the form [12]

$$
G_{A}=4 \pi g u_{x}\left(\frac{\pi}{2}\right)
$$

and

$$
\begin{aligned}
\left\langle I_{\omega} I_{-\omega}\right\rangle & =\frac{e^{2} G_{A}}{3 \hbar}\left\{\left(3-P_{S}\right) \Psi(\omega)\right. \\
& \left.+\frac{1}{2} P_{S}[\Psi(\omega-2 e V)+\Psi(\omega+2 e V)]\right\},
\end{aligned}
$$

where $\Psi(\omega)=\omega \operatorname{coth}(\omega / 2 T)$, and the superconductive noise function $P_{S}$ is given by

$$
P_{S}=1-\frac{u_{x x x}\left(\frac{\pi}{2}\right)}{2 u_{x}\left(\frac{\pi}{2}\right)} .
$$


The quantity $F=(2 / 3) P_{S}$ gives the reduction of the shot noise between the normal and superconducting terminals, $\langle I I\rangle_{\text {shot }}=F e\langle I\rangle$, compared to its Poissonian value for a single electron tunneling, and can be identified with the Fano factor [25].

For an N-S system, all quantities are expressed in terms of derivatives of $u(x)$ at $x=\pi / 2$. The counterparts of Eqs. (14)-(6) for the case when the $\mathrm{S}$ island is in the normal state can also be expressed in terms of the same function $u(x)$, but with derivatives taken at $x=0$. The conductance of the system $G=4 \pi g u_{x}(0)$, while the current-current correlator is given by Eq. (5) with the replacements $G_{A} \rightarrow G, 2 \mathrm{eV} \rightarrow \mathrm{eV}$, and $P_{S} \rightarrow P_{N}=$ $1-2 u_{x x x}(0) / u_{x}(0)$.

In principle, one can go further and calculate the full charge transfer statistics in terms of the function $u(x)$ following the derivation 14, 15] in the scattering matrix technique.

\section{FUNCTIONAL RG}

We have seen that the set $\left\{\gamma_{n}\right\}$ or, equivalently, the function $u(x)$ encodes all information about charge transmission through the system. Now we will discuss how to determine the charges $\gamma_{n}$ for the system shown in Fig. 1. 1 .

The starting point is the microscopic Keldysh action for a $\mathrm{S}$ island in contact with a disordered metal, derived in Ref. 13. It can be represented as a sum of the bulk and boundary contributions. The bulk action, $S_{\text {bulk }}$, is a functional of three fluctuating fields: the matter field $Q\left(\mathbf{r}, t, t^{\prime}\right)$ in the film, the electromagnetic potential, and the order parameter field used to decouple the quartic interaction vertex in the Cooper channel. $Q\left(\mathbf{r}, t, t^{\prime}\right)$ is a matrix in the direct $4 \times 4$-dimensional product $K \otimes N$ of the Keldysh and Nambu-Gor'kov spaces, its average value giving the electron Green function in the Keldysh form, $\hat{G}(\mathbf{r}, \mathbf{r})$. In the tunneling Hamiltonian approximation, the boundary action,

$$
S_{\mathrm{tun}}\left[Q_{S}, Q\right]=-\frac{i \pi G_{T}}{4} \operatorname{Tr} Q_{S} Q(d),
$$

describes an elementary tunneling process between the $\mathrm{S}$ island and the $\mathrm{N}$ metal. Here $G_{T}=\hbar / e^{2} R_{T}$ is the dimensionless tunneling conductance of the interface, and $Q(d)$ is taken at the metal side of the N-S boundary.

The next step is to eliminate degrees of freedom in the $\mathrm{N}$ film. 2D geometry of the system suggests that this procedure can be realized with the help of the Renormalization Group (RG) approach by successive integrating over fast modes in the bulk. As a result, the boundary action (7) which initially described single Andreev reflection process gets modified by multiple Andreev reflections and acquires the form

$$
S_{\mathrm{bound}}=-i \pi^{2} g \sum_{n=1}^{\infty} \gamma_{n}(\zeta) \operatorname{Tr}\left[Q_{S} Q(r)\right]^{n} .
$$

where the logarithmic variable $\zeta=2 \ln (r / d)=\ln \left(\omega_{d} / \Omega\right)$, $\omega_{d}=D / d^{2}$ is the high-frequency cutoff, and $\Omega=D / r^{2}$ is the energy associated with the current scale $r$. At the energy scale $\omega_{d}$, the multicharge action (8) reduces to Eq. (7): $\gamma_{1}(0)=a \equiv G_{T} / 4 \pi g$, and $\gamma_{n \geq 2}(0)=0$, and, consequently, $u(x, \zeta=0)=a \sin x$.

Under the action of the RG, the charges $\gamma_{n}(\zeta)$ are changing. In the zero-energy limit, when the Cooperon decoherence energy $\Omega_{*}$ is smaller than the current RG energy scale $\Omega$, both diffusons and Cooperons contribute to the RG equation which, written in terms of the function $u(x, \zeta)$, reads 12 .

$$
u_{\zeta}+u u_{x}=-\lambda(\zeta) u\left(\frac{\pi}{2}, \zeta\right) \sin x .
$$

The r.h.s. of the functional RG equation (9) is due to the Cooper channel interaction with the scale-dependent constant $\lambda(\zeta)$ given by [17, 13]

$$
\lambda(\zeta)=\frac{\lambda_{d}+\lambda_{g} \tanh \lambda_{g} \zeta}{1+\frac{\lambda_{d}}{\lambda_{g}} \tanh \lambda_{g} \zeta},
$$

where $\lambda_{d}$ is defined at the energy scale $\omega_{d}$. At $\zeta>\sqrt{g}$, Coulomb repulsion in 2D drives $\lambda(\zeta)$ toward the Fikelstein's fixed point $\lambda_{g}=1 / 2 \pi \sqrt{g}$. In deriving Eq. (9) we neglected the weak localization and interaction corrections to the film conductance $g$, making use of the small parameter $\zeta / g \ll 1$.

In the absence of Cooper channel interaction, Eq. (9) acquires the form of the Euler equation. In this case it is convenient to rewrite it in terms of the new variable $t=R_{D} / R_{T}=a \zeta$ and the function $U(x, t)=u(x, \zeta) / a$ as $U_{t}+U U_{x}=0$, with the initial condition $U(x, 0)=\sin x$. In this representation, all information about geometry of the system had gone, and the Euler equation for $U(x, t)$ describes evolution of $\left\{\gamma_{n}\right\}$ for any (not only 2D) system consisting of a tunnel barrier and a diffusive conductor in the zero-energy limit 223,26, 12].

If the decoherence energy scale $\Omega_{*}>E_{\mathrm{Th}}$ then Cooperons become inoperative at the latest stage of renormalization procedure, where $\zeta>\zeta_{*} \equiv \ln \left(\omega_{d} / \Omega_{*}\right)$. In this case the functional RG equation reduces to

$$
\tilde{u}_{\zeta}+\tilde{u} \tilde{u}_{x}=0,
$$

where $\tilde{u}(x)=[u(x)-u(\pi-x)] / 2$. The function $\tilde{u}\left(x, \zeta ; \zeta_{*}\right)$ bears the knowledge of the decoherence scale $\Omega_{*}$ through the initial condition $\tilde{u}\left(x, \zeta_{*} ; \zeta_{*}\right)=\left[u\left(x, \zeta_{*}\right)-u(\pi-\right.$ $\left.\left.x, \zeta_{*}\right)\right] / 2$, where $u\left(x, \zeta_{*}\right)$ is the solution of Eq. (9) at the upper border of its applicability, $\zeta=\zeta_{*}$. Note, however, that crossover from the $\zeta<\zeta_{*}$ to $\zeta>\zeta_{*}$ regimes is described by Eqs. (9), (11) with the logarithmic accuracy only. 
The RG equations (9) and (11) should be integrated until we reach the perfect boundary between the $\mathrm{N}$ film and the reservoir. Then, substituting $Q(L)=Q_{N}$ into the effective boundary action (8), we arrive at the Proximity Action (11) with $\gamma_{n}=\gamma_{n}\left(\zeta_{\mathrm{Th}}\right)$, where $\zeta_{\mathrm{Th}}=$ $\ln \left(\omega_{d} / E_{\mathrm{Th}}\right)=2 \ln (L / d)$.

\section{ANDREEV CONDUCTANCE}

Now we proceed with the analysis of the functional RG equations derived in the previous section. We start from the zero-energy limit, $\Omega_{*}<E_{\mathrm{Th}}$, described by Eq. (9). In the absence of interaction one readily reproduces the known result [3,4] for the Andreev conductance:

$$
G_{A}^{(0)}=G_{T} \frac{\sin \Theta(t)}{1+t \sin \Theta(t)}=G_{D} \frac{t \sin \Theta(t)}{1+t \sin \Theta(t)},
$$

where $t=a \zeta_{\mathrm{Th}}=R_{D} / R_{T}, R_{D}=\ln (L / d) / 2 \pi \sigma$, and the function $\Theta(t)$ satisfies $\Theta(t)=t \cos \Theta(t)$.

It is often convenient to characterize the system with the help of the effective interface resistance $R_{T \text {,eff }}$ defined formally through $R_{A}=R_{D}+R_{T \text {,eff }}$. For a noninteracting system, the ratio $R_{T, \text { eff }} / R_{T}$ as a function of $t=R_{D} / R_{T}$ is shown in Fig. 2 by the dashed line. It behaves as $t^{-1}$ for $t \ll 1$ and saturates at 1 for $t \gg 1$. Correspondingly, $G_{A}^{(0)}=G_{T}^{2} / G_{D}$ for $t \ll 1$, and $G_{A}^{(0)}=G_{D}$ for $t \gg 1$.

At relatively small scales, the effect of Cooper interaction described by the r.h.s. of Eq. (9) can be considered perturbatively. The magnitude of the first-order interaction correction to the quasiclassical conductance (12), $\delta G_{A} \sim-\lambda \zeta G_{A}^{(0)}$, grows [12] with the system size $L$ and eventually becomes of the order of $G_{A}^{(0)}$ at $\zeta \sim 1 / \lambda$. Comparing this scale to the scale $\zeta \sim 1 / a$, corresponding to the crossover from the tunneling $(t \ll 1)$ to diffusive $(t \gg 1)$ limits, one can distinguish between the regimes of strong, $\lambda \gg a \equiv G_{T} / 4 \pi g$, and weak, $\lambda \ll a$, repulsion (still, in both cases $\lambda \ll 1$ ).

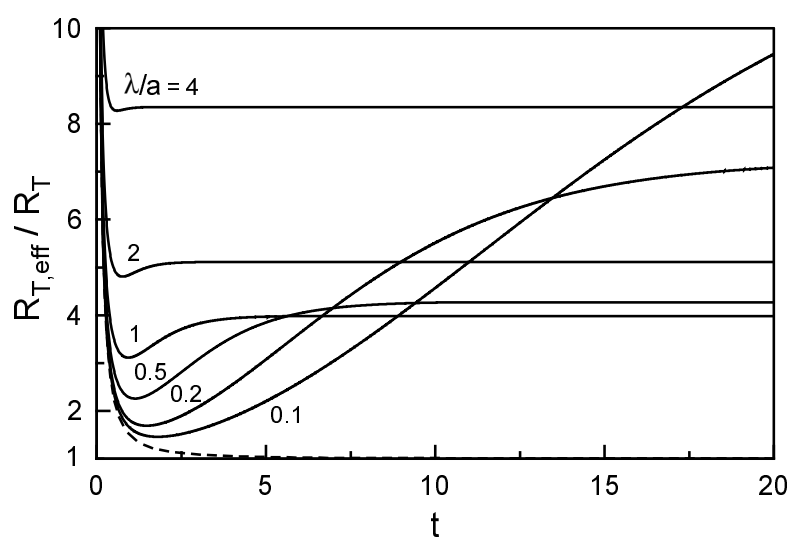

FIG. 2. Dependence of the effective interface resistance $R_{T, \text { eff }}(t) / R_{T}$ vs. $t=R_{D} / R_{T}$ for different values of $\lambda / a$ obtained by numerical solution of Eq. (9) for $\lambda(\zeta)=$ const. The dashed line corresponds to the noninteracting case, $\lambda=0$.
In general, the functional RG equation (9) does not allow an analytical solution, and should be solved numerically. For clarity, we will assume hereafter that the interaction constant had reached the Finkelstein's fixed point $\lambda_{g}=1 / 2 \pi \sqrt{g}$, so that strong repulsion corresponds to a relatively weak tunneling conductance $G_{T} \ll 2 \sqrt{g}$, and vice versa. The effective interface resistance $R_{T \text {,eff }}$ normalized to the tunneling resistance $R_{T}$ as a function of $t=R_{D} / R_{T}$ is plotted in Fig. 2 for different values of the ratio $\lambda_{g} / a$. For the case of strong repulsion, $\lambda_{g} \gg a, R_{T, \text { eff }}(t)$ very quickly (at $t \sim a / \lambda_{g} \ll 1$ ) reaches its asymptotic value and saturates at $R_{T, \text { eff }}(t=\infty) \approx$ $\left(2 \lambda_{g} / a\right) R_{T}$. The limiting value $R_{T, \text { eff }}(\infty)$ decreases with the decrease of $\lambda_{g} / a$ up to $\lambda_{g} / a \sim 1$. At smaller $\lambda_{g} / a$, corresponding to the case of weak repulsion, $R_{T, \text { eff }}(\infty)$ starts to grow again, and reaches an asymptotic behavior $R_{T, \text { eff }}(\infty) / R_{T} \approx 1.19 a / \lambda_{g}$ at $\lambda_{g} / a \ll 1$. In this limit $R_{T, \text { eff }}(t)$ reaches its asymptotic value at large scale $t \sim a / \lambda_{g}$.

The data of Fig. 2 demonstrate that in a $2 \mathrm{D}$ system the limits $\lambda \rightarrow 0$ and $R_{D} \rightarrow \infty$ do not commute: for any small but finite $\lambda_{g}, R_{T \text {,eff }}(t) / R_{T}$ will eventually (though, at very large $t$ ) deviate from the noninteracting dependence (dashed line in Fig. 2) and become large.

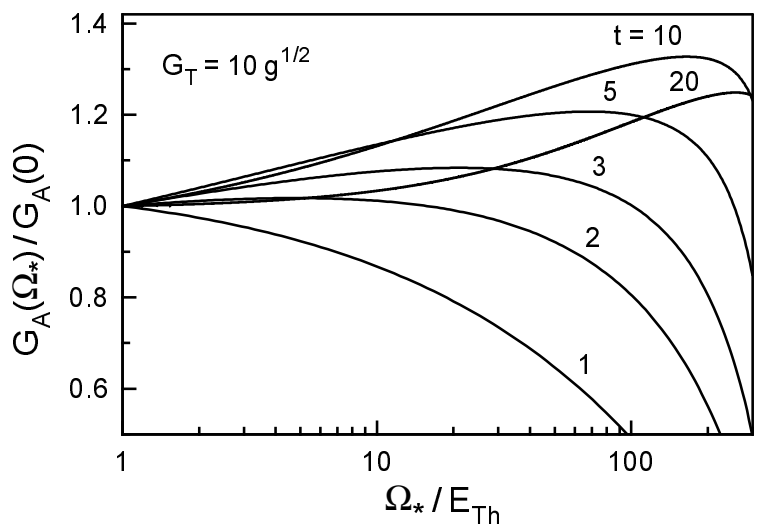

FIG. 3. Dependence of the Andreev conductance $G_{A}\left(\Omega_{*}\right)$ (normalized to the zero-energy value) on the ratio $\Omega_{*} / E_{\mathrm{Th}}$ for different values of $t$. For all plots, $\omega_{d} / E_{\mathrm{Th}}=400, \lambda=\lambda_{g}$, and $G_{T}=10 \sqrt{g}$.

At large enough temperature, voltage, or magnetic field, $\Omega_{*} \equiv \max (T, e V, e D H / c) \gg E_{\mathrm{Th}}$, the resistance of the structure is given by [12]

$$
R_{A}\left(t, t_{*}\right)=R_{D}+R_{T, \mathrm{eff}}\left(t_{*}\right)
$$

where $t_{*}=a \zeta_{*}=\left(G_{T} / 4 \pi g\right) \ln \left(\omega_{d} / \Omega_{*}\right)$. Therefore, a nonmonotonous behavior of $R_{T \text {,eff }}\left(t_{*}\right)$ will manifest itself in a nonmonotonous temperature, voltage and magnetic field dependence of the subgap conductance. The unusual enhancement of conductivity with the increase of the decoherence energy scale $\Omega_{*}$ is most pronounced in 
the limit of weak repulsion, $G_{T} \gg 2 \sqrt{g}$. Since the total resistance is the sum of $R_{T \text {,eff }}$ and $R_{D}$, cf. Eq. (13), the magnitude of the effect is determined by the ratio $R_{T, \text { eff }} / R_{D}$ which has a maximum at $t \approx G_{T} / \sqrt{g}$. An example of such a nonmonotonous dependence of $G_{A}\left(\Omega_{*}\right)$ is shown in Fig. 3. The curves differ by the ratio $t=R_{D} / R_{T}$ and correspond to $G_{T}=10 \sqrt{g}$ (i. e., $\left.\lambda_{g} / a=0.2\right)$.

We emphasize that the described nonmonotonous behavior has nothing to do with usual "finite-bias" conductance anomaly in N-S structures with good interfaces [27,11], which occur at $(T, e V) \approx E_{\mathrm{Th}}$ even in the absense of interaction in the $\mathrm{N}$ region. In simple terms the origin of this new effect can be understood as follows: repulsion in the normal metal produces a superconductive "gap function" in the normal conductor, $\Delta_{N}$, with the negative (compared to $\Delta_{S}$ in a superconductor) sign. Due to its opposite sign, $\Delta_{N}$ decreases the conductance of the structure, therefore any decoherence that reduces $\Delta_{N}$ leads to the increase of the conductance.

\section{NOISE}

Now we turn to the analysis of the noise function $P_{S}$. For a noninteracting system in the zero-energy limit it is given by [28, 12 .

$$
P_{S}^{(0)}(t)=1+\frac{1+\Theta(t) \tan \Theta(t)+3 \Theta(t) \cot \Theta(t)}{2[1+\Theta(t) \tan \Theta(t)]^{4}} .
$$

Eq. 14) describes a crossover from the Poissonian $\left(P_{S}=\right.$ 3) to the sub-Poissonian $\left(P_{S}=1\right)$ [29] character of the noise as the system evolves from the tunnel $(t \ll 1)$ to the diffusive $(t \gg 1)$ limits.

In the presence of interaction, $P_{S}$ can be estimated, qualitatively, by comparing the effective tunneling resistance, $R_{T, \text { eff }}$, with the diffusive resistance, $R_{D}$. For $R_{T, \text { eff }} \gg R_{D}, P_{S} \approx 3$, while in the opposite case $P_{S} \approx 1$. If we again assume that $\lambda(\zeta)=\lambda_{g}$, then $P_{S}$ will become a function of two parameters: $t=R_{D} / R_{T}$ and $\lambda_{g} / a=2 \sqrt{g} / G_{T}$. The boundaries between the regions with $P_{S}=3$ and $P_{S}=1$ on the plane $\left(\log t, \log \left(G_{T} / \sqrt{g}\right)\right)$ are sketched in Fig. 4 .

In the case of strong repulsion, $G_{T} \ll \sqrt{g}$, $R_{T, \text { eff }}$ very quickly (at $t \sim G_{T} / \sqrt{g}$ ) saturates at $R_{T, \text { eff }}(\infty) \equiv\left(4 \sqrt{g} / G_{T}\right) R_{T}$. Then one obtains [12] $P_{S}^{\text {int }}=$ $P_{N}\left[R_{D} / R_{T, \text { eff }}(\infty)\right]$, where $P_{N}(t)=1+2 /(1+t)^{3}$ is the noise function [3,12] for the system with the $\mathrm{N}$ island. Thus, in the limit $G_{T} \ll \sqrt{g}$, the crossover between the tunnel and diffusive character of noise is shifted to $t \sim \sqrt{g} / G_{T} \gg 1$, see Fig. 目.

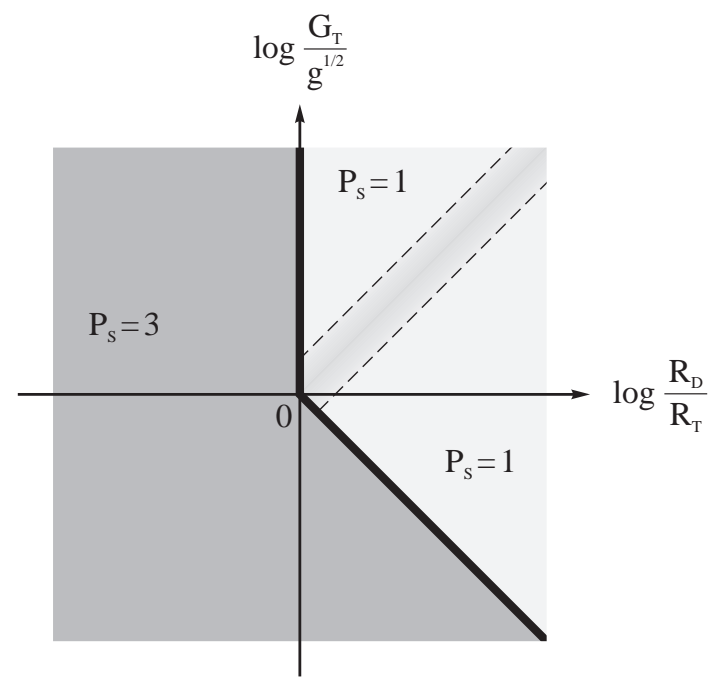

FIG. 4. Schematic map of the noise coefficient $P_{S}$ as a function of the tunnel and diffusive resistances in the presence of Cooper repulsion $\lambda=\lambda_{g}$. Dark area refers to the tunnel limit $P_{S} \approx 3$, whereas light regions correspond to the diffusive regime with $P_{S} \approx 1$.

In the limit of weak repulsion, $G_{T} \gg \sqrt{g}$, the situation is more interesting. For $t \sim 1$, interaction corrections can be neglected and $P_{S}$ is given by the noninteracting expression (14). So, at $t \sim 1, P_{S}$ decreases from 3 to 1 , the corresponding boundary being shown in Fig. 4 . Later, at $t \sim G_{T} / \sqrt{g} \gg 1$ (when $R_{D} \sim \hbar / e^{2} \sqrt{g}$ ) interaction corrections become relevant. In this region, $R_{T \text {,eff }}$ is of the order of $R_{D}$, and one may anticipate that $P_{S}$ will deviate from 1 . For even larger $t$ when resistance is dominated by the diffusive conductor, $P_{S}$ will eventually reduce down to 1 . This crossover region is marked in Fig. 1 by the dashed lines. The function $P_{S}$ in the crossover region $t \sim G_{T} / \sqrt{g} \gg 1$ obtained by numerical solution of Eq. (9) is plotted in Fig. 5 as a function of $s=\lambda_{g} \zeta=(\ln L / d) / \pi \sqrt{g}=2\left(e^{2} / \hbar\right) R_{D} \sqrt{g}$. It has a minimum $P_{S}=0.99$ at $s=0.40$ and a maximum $P_{S}=1.28$ at $s=3.25$.

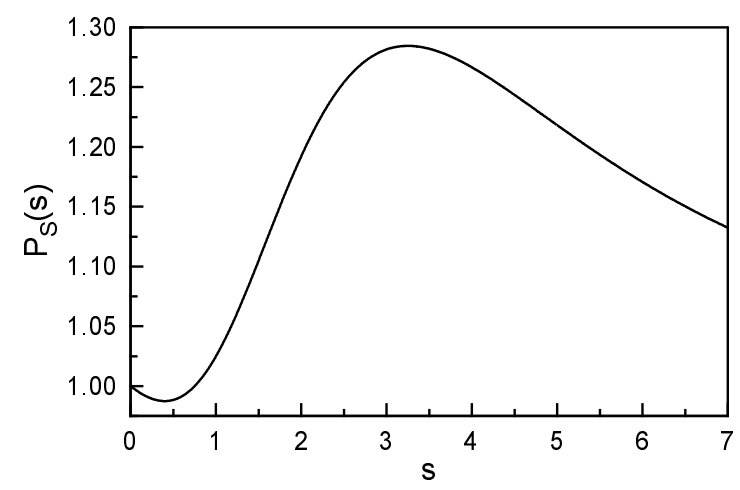

FIG. 5. Noise function $P_{S}(s)$ vs. $s=2 \sqrt{g} / G_{D}$ for the case of weak interaction, $G_{T} \gg \sqrt{g}$. 
Finally, we consider the $\Omega_{*}$ dependence of the noise power. In the case of weak interaction, $P_{S}$ decreases (at $t, t_{*} \gg 1$ ) with the increase of the Cooperon decoherence energy scale $\Omega_{*}$, as if the system is becoming more diffusive. This trend is opposite to what one has in the noninteracting case when the increase of $\Omega_{*}$ drives the system toward the tunnel limit, thus, increasing $P_{S}$. In the limit of strong repulsion, $G_{T} \ll \sqrt{g}$, the zeroenergy noise function $P_{S}(t)$ exhibits a crossover from the tunnel to diffusive regimes at $t \sim \sqrt{g} / G_{T} \gg 1$. Nevertheless, upon increase of $\Omega_{*}$ the function $P_{S}\left(t, t_{*}\right)$ remains $t_{*}$ independent down to much smaller values of $t_{*} \sim G_{T} / \sqrt{g} \ll 1$ which correspond to the energy scales $\ln \left(\omega_{d} / \Omega_{*}\right) \simeq 2 \pi \sqrt{g}$.

\section{CONCLUSION}

We developed the Proximity Action functional method able to describe quantum charge transport in mesoscopic superconductive structures in the presence of interaction in the normal metal. New method is applied to the study of the effect of repulsion in the Cooper channel upon Andreev conductance and noise in $2 \mathrm{D} \mathrm{N}-\mathrm{S}$ structures. Interaction corrections scale as $g^{-1 / 2} \ln (L / d)$ and lead to nonmonotonous dependence of both the conductance and the Fano factor upon temperature, voltage and magnetic field.

\section{ACKNOWLEDGMENTS}

We are grateful to G. B. Lesovik and Yu. V. Nazarov for useful discussions. This research was supported by the NSF grant DMR-9812340 (A. I. L.), RFBR grant 9802-16252, NWO-Russia collaboration grant, Swiss NSFRussia collaboration grant 7SUPJ062253.00, and by the Russian Ministry of Science via the project "Mesoscopic electron systems for quantum computing" (M. V. F. and M. A. S.).

[1] A. F. Andreev, Zh. Eksp. Teor. Fiz. 46, 1823 (1964) [Sov. Phys. JETP 19, 1228 (1964)].

[2] A. F. Volkov, A. V. Zaitsev and T. M. Klapwijk, Physica C210, 21 (1993).
[3] Yu. V. Nazarov, Phys. Rev. Lett. 73, 134 (1994).

[4] Yu. V. Nazarov, Phys. Rev. Lett. 73, 1420 (1994).

[5] C. W. J. Beenakker, Rev. Mod. Phys. 69, 731 (1997).

[6] B. Pannetier and H. Courtois, cond-mat/9912024.

[7] A. I. Larkin and Yu. N. Ovchinnikov, in Nonequilibrium Superconductivity, eds. D. N. Langenberg and A. I. Larkin (Elsevier, New York, 1986).

[8] C. J. Lambert, J. Phys. Condens. Matter 3, 6579 (1991); C. W. J. Beenakker, Phys. Rev. B 46, 12841 (1992).

[9] B. L. Altshuler and A. G. Aronov, in Electron-Electron Interactions in Disordered Conductors, ed. A. J. Efros and M. Pollack, Elsevier Science Publishers, NorthHolland, 1985.

[10] F. Zhou, B. Z. Spivak and A. Yu. Zyuzin, Phys. Rev. B 52, 4467 (1995).

[11] T. H. Stoof and Yu. V. Nazarov, Phys. Rev. B 53, 14496 (1996).

[12] M. A. Skvortsov, A. I. Larkin, and M. V. Feigel'man, cond-mat/0008463.

[13] M. V. Feigel'man, A. I. Larkin, and M. A. Skvortsov, Phys. Rev. B 61, 12361 (2000).

[14] L. S. Levitov and G. B. Lesovik, Pis'ma Zh. Eksp. Teor. Phys. 58, 225 (1993) [JETP Lett. 58, 230 (1993)].

[15] H. Lee, L. S. Levitov, and A. Yu. Yakovets, Phys. Rev. B 51, 4079 (1995).

[16] A. M. Finkelstein, Pis'ma ZhETF 45, 37 (1987) [JETP Letters 45, 46 (1987)].

[17] A. M. Finkelstein, Electron Liquid in Disordered Conductors, Vol. 14 of Soviet Scientific Reviews, ed. I. M. Khalatnikov, Harwood Academic Publishers, London, 1990.

[18] A. M. Finkelstein, Physica B 197, 636 (1994).

[19] E. Abrahams, P. W. Anderson, D. C. Licciardello and T. V. Ramakrishnan, Phys. Rev. Lett. 42, 673 (1979).

[20] L. P. Gorkov, A. I. Larkin and D. E. Khmelnitsky, Pis'ma ZhETF 30, 248 (1979) [JETP Letters 30, 228 (1979)].

[21] B. L. Altshuler, A. G. Aronov and P. A. Lee, Phys. Rev. Lett. 44, 1288 (1980).

[22] L. S. Levitov and A. V. Shytov, Pis'ma ZhETF 66, 200 (1997) [JETP Letters 66, 214 (1997)].

[23] C. W. J. Beenakker, B. Rejaei, and J. A. Melsen, Phys. Rev. Lett. 72, 2470 (1994).

[24] M. V. Feigel'man, A. I. Larkin, and M. A. Skvortsov, cond-mat/0010402.

[25] Ya. M. Blanter and M. Büttiker, Phys. Rep. 336, 1 (2000).

[26] Yu. V. Nazarov, in Quantum Dynamics of Submicron Structures, eds. H. Cerdeira, B. Kramer, G. Schoen (Kluwer, 1995); cond-mat/9410011.

[27] S. N. Artemenko, A. F. Volkov and A. V. Zaitsev, Solid State Commun. 30, 771 (1995).

[28] M. J. M. de Jong and C. W. J. Beenakker, Phys. Rev. B 49, 16070 (1994).

[29] C. W. J. Beenakker, M. Büttiker, Phys. Rev. B 46, 1889 (1992); K. E. Nagaev, Phys. Lett. A 169, 103 (1992). 